\title{
Crisis económica y salud: lecciones aprendidas y recomendaciones para el futuro*
}

\author{
Juan Oliva \\ Luz María Peña-Longobardo \\ Universidad de Castilla-La Mancha \\ Beatriz González López-Valcárcel \\ Patricia Barber Pérez \\ Universidad de Las Palmas de Gran Canaria \\ Néboa Zozaya González \\ Fundación Weber
}

\section{Resumen}

La reciente crisis económica vivida en España, pese al sufrimiento y pérdida de bienestar ocasionadas, no parece haber afectado de una manera severa, al menos a corto plazo, a la mortalidad y la salud autopercibida de la población general. No obstante, sí se ha producido un impacto negativo sobre la salud mental, especialmente entre los varones. Asimismo, hay grupos vulnerables de población cuya salud ha empeorado durante este periodo, intensificándose las desigualdades sociales en la misma. Dada la alarmante evolución observada en los indicadores sociales de desigualdad de rentas y tasas de pobreza y riesgo de exclusión social, conviene ser cautos con los resultados identificados en el corto plazo y vigilantes sobre la evolución de la salud de la población en el medio y largo plazo, a la vez que se extraen las conclusiones oportunas sobre las políticas e intervenciones a implementar en futuras situaciones de crisis.

Palabras clave: crisis, salud, España.

Clasificación JEL: II, II80.

\begin{abstract}
Even though the considerable loss of welfare caused by the recent economic crisis occurred in Spain, it does not seem to have affected heavily, at least in the short term, in terms of mortality and self-perceived health of the general population. However, there has been a negative impact on mental health, especially among men. Likewise, there are several vulnerable population groups whose health has worsened during this period, intensifying social inequalities in health. Given such disturbing evolution observed in the social indicators of income inequality and poverty rates as well as risk of social exclusion, caution is needed when interpreting the results obtained in the
\end{abstract}

* Este trabajo se ha elaborado a partir de un texto previo sobre el mismo tema encargado a los autores por el Ministerio de Sanidad. Deseamos agradecer el apoyo y la ayuda recibidos por Mercedes Alfaro y Rosa María Urbanos. Asimismo, agradecemos los comentarios recibidos por parte de Jordi Alonso, Enrique Bernal, José María Labeaga, Soledad Márquez, Fernando Rodríguez Artalejo, Marc Sáez y María Luisa Vázquez. Los errores y omisiones son responsabilidad exclusiva de los autores. 
short term and attention should be paid on the population's health evolution in the medium and long term. At the same time, it is necessary to draw the appropriate conclusions so that the policies and interventions could be implemented in future crises.

Keywords: crisis, health, Spain.

JEL classification: I1, I180.

\section{Introducción}

La crisis financiera desencadenada en Estados Unidos a finales de 2007 y transmitida a otros países, devino en una crisis económica a gran escala que estalló con virulencia inusitada en el año 2008. Crisis en tres actos bien diferenciados: crisis financiera y pérdida de confianza en el sistema, parálisis acentuada del crédito y crisis de las deudas soberanas. Llegados a este punto, el discurso de refundar el capitalismo ya había sido olvidado y la respuesta de los gobiernos europeos en esta fase fue una fuerte restricción de las cuentas públicas bajo el auspicio de la austeridad. La crisis se caracterizó también por su asimetría, tanto a nivel mundial como europeo, donde algunos países, como España, se vieron especialmente comprometidos.

La crisis ha afectado de manera intensa a la economía y la sociedad española y ha generado un enorme sufrimiento a la población, empobreciendo a muchas familias y sumiéndolas en la inseguridad. Aunque oficialmente dejáramos atrás la crisis en términos macroeconómicos en el año 2014, el nivel de PIB del año 2007 no se superó hasta 2016 y el máximo de 2008 no se volvió a alcanzar hasta el primer trimestre de 2017, junto a una herencia adquirida de una deuda pública cercana al 100 por 100 del PIB. A nivel social, vistos los indicadores actuales (verano de 2018) de desigualdad en renta y el porcentaje de población en riesgo de pobreza o de exclusión social, es mucho más discutible afirmar que la crisis ha quedado atrás. Así pues, la crisis no solo ha dejado cicatrices a la sociedad, sino que la recuperación económica no ha restaurado los niveles iniciales de muchos indicadores sociales, por lo que sus efectos podrían prolongarse durante años.

La salud es uno de los pilares donde se apoya el bienestar de los individuos y las sociedades. Desde la perspectiva individual, aunque disfrutar de una buena salud no es condición suficiente para alcanzar o mantener un alto grado de bienestar, en términos generales, es condición necesaria, como lo muestran distintos estudios sobre felicidad y satisfacción con la vida realizados en las últimas décadas (Graham, 2008). En este contexto cabe preguntarse cuál ha sido el efecto de la crisis sobre la salud de las personas que residen en España. Aunque puede resultar de interés explorar los efectos de la crisis sobre la sanidad y la utilización de servicios sanitarios, y esta línea es la que más abunda en los medios de comunicación y en el imaginario colectivo, el foco de este artículo se dirige al resultado final, esto es, la salud de la población, y no a los inputs intermedios -sanidad, gasto público.

El trabajo se articula de la siguiente manera. Tras esta breve introducción, revisaremos los diferentes tipos de efectos y mecanismos de acción de las crisis 
económicas sobre la salud y el papel de las políticas públicas, identificando qué grupos de población han sido señalados como más vulnerables en crisis anteriores, así como las dificultades metodológicas más relevantes para identificar los efectos de las crisis y el conocimiento acumulado de situaciones precedentes. A continuación, se lleva a cabo una revisión de la literatura generada en España o sobre España en relación a los efectos que ha tenido la crisis sobre la mortalidad, salud autopercibida, salud mental y problemas de salud en grupos seleccionados. Finalmente, el artículo se cierra con las conclusiones obtenidas de los apartados previos y con una serie de recomendaciones dirigidas a los responsables públicos sobre políticas en salud.

\section{2. ¿Qué sabemos acerca de los mecanismos y efectos de las crisis económicas del pasado sobre la salud?}

\subsection{Mecanismos, efectos y poblaciones más afectadas}

Las crisis económicas suponen riesgos para la salud de la población, alterando los recursos disponibles para la ejecución de políticas públicas (incluyendo las sanitarias, pero no únicamente), destruyendo empleo, reduciendo las rentas familiares y generando migraciones masivas. No obstante, no hay leyes universales sobre las reacciones de la salud ante las crisis, dado que dependen del contexto institucional, cultural y social y de la intensidad, duración y velocidad de la caída, así como de los tipos de respuestas públicas y sociales. Por tanto, los efectos de las crisis dependen en gran medida de las políticas de protección social, de la red de seguridad del Estado del Bienestar y de la vertebración de las redes sociales y familiares. De este modo, las crisis que han tenido lugar en los mismos años han afectado de manera dispar a distintos países en función de los elementos señalados.

Los efectos de corto plazo de las crisis sobre la salud se pueden producir por tres vías, muy relacionadas entre sí: las migraciones, el empobrecimiento y el desempleo. Las migraciones masivas son la respuesta a la pobreza y al empobrecimiento causado en múltiples ocasiones por las crisis económicas. El efecto renta de las crisis depende del nivel de partida y de llegada y de la velocidad del cambio. Las relaciones entre renta y salud, bidireccionales y no lineales, cambian a lo largo de la vida de las personas, pero los niños pobres son particularmente vulnerables. La pérdida de renta de las familias y su rápido empobrecimiento durante las crisis se encuentran entre las causas de mayor relevancia. En la mayoría de las ocasiones, las caídas de renta son consecuencia de la pérdida del empleo y la imposibilidad de encontrar otro trabajo. Cuando se cae por debajo de cierto umbral crítico de recursos familiares, ello puede derivar en un deterioro de la salud, incluso a corto plazo. Una de las consecuencias inmediatas del empobrecimiento es el cambio de pautas de alimentación, que, dada la estructura de precios relativos de los alimentos, puede llevar, paradójicamente, a la obesidad de las familias empobrecidas del primer mundo (Drewnowski y Specter, 2004). La pobreza y la falta de educación son «causas» de la obesidad, y la calidad 
de la dieta está muy relacionada con el nivel socioeconómico (Darmon y Drewnowski, 2008).

Por otro lado, el sector público tiene un papel fundamental en las crisis, no solo por las políticas, sanitarias o no, que afectan directa o indirectamente a la salud, sino también por su capacidad para distribuir en el tiempo sus efectos económicos -planes de inversión, déficit corriente para afrontar las políticas sociales y redistributivas. Así pues, la propia política económica tiene la capacidad de agudizar o aminorar el empeoramiento de la salud si retrasa o acelera la salida de la crisis. Además de los efectos más visibles a corto plazo, como la reducción general de los presupuestos públicos, un efecto indirecto de las crisis es que la salud pierde prioridad para los gobiernos y visibilidad para las poblaciones, ante los problemas económicos más acuciantes. Se reducen los presupuestos de salud y de asistencia sanitaria, y se demoran los planes de inversión, incluyendo los de capital humano. Junto a ello, ante la urgencia de la crisis, se corre el riesgo de perder la atención a la intersectorialidad, a la «salud en todas las políticas» y a la prevención y los programas de largo plazo.

Las experiencias de crisis anteriores nos enseñan que algunas políticas han amortiguado y acortado los efectos adversos de las crisis económicas. En efecto, las redes sociales individuales y el mantenimiento de las políticas sociales actúan como factores protectores de la salud en esos periodos (Rivadeneyra-Sicilia et al., 2014). Además, los programas y políticas laborales activas que mantienen y reintegran trabajadores al mercado podrían mitigar los efectos adversos del desempleo sobre la salud que se producen durante las recesiones económicas (Stuckler et al., 2009a). Según la corriente dominante durante esta crisis en la literatura biomédica, las políticas del bienestar y el mantenimiento del gasto social podrían ser potentes antídotos contra los efectos adversos de la recesión mientras que, por el contrario, las políticas restrictivas del gasto público y el objetivo de control del déficit serían nocivos para la salud. Este mensaje se ha plasmado en una frase de alto impacto mediático: «La austeridad mata» (Stuckler y Basu, 2013). Una palabra (austeridad) que tradicionalmente tenía connotaciones positivas, queda denostada y adquiere un tono peyorativo dominante en esos años (Segura, 2014). Pero la base empírica del «austericidio» es con frecuencia endeble, porque infiere resultados generales del análisis de entornos concretos (por ejemplo, comparando Grecia, Portugal y España con Islandia; Karanikolos et al., 2013) donde la crisis no había hecho más que empezar (Stuckler et $a l ., 2009$ a) y los gobiernos apenas habían empezado a definir sus reacciones de restricción o mantenimiento del gasto. Pero ni los datos ni los métodos de esos estudios carecen de problemas. Aunque a veces los autores previenen de la dificultad para estimar relaciones causa-efecto con datos ecológicos (Baumbach y Gulis, 2014), lo cierto es que en muchas ocasiones concluyen como si dichas relaciones hubieran sido identificadas en el curso de sus trabajos.

Por tanto, en cualquier caso, el análisis de las políticas públicas durante, y como reacción a, las situaciones de crisis económicas ha de considerar las distintas dimensiones del bienestar social, e ir más allá de las intervenciones en el sistema sanitario 
que, si bien son las más visibles ${ }^{1}$, no necesariamente han de coincidir con las de mayor impacto sobre la salud. Algunas de las lecciones sobre políticas extraídas de las crisis de los años 90 del siglo pasado se basan precisamente en plantear simultáneamente políticas macroeconómicas y atención a los mercados junto a medidas de protección social a los más vulnerables (Stiglitz, 1999).

Otro punto a considerar es identificar a qué grupos de población afectan más las crisis económicas. Una crisis económica como la vivida en España, que no desplomó el PIB pero lo redujo significativamente, y que contrajo sensiblemente el gasto sanitario público y las políticas sociales, puede no notarse a corto plazo en los indicadores de salud de la población general, pero sí en determinados grupos vulnerables: niños, ancianos, pobres, desempleados e inmigrantes. La infancia merece interés especial, porque las consecuencias de las privaciones en la infancia se harán notar a lo largo de toda la vida. La pobreza se transmite entre generaciones (Case y Paxson, 2002) y la mala salud desempeña un papel importante en esa transmisión. Así, los niños de familias pobres pueden contraer enfermedades crónicas que reducen su capital humano en la edad adulta. La baja renta de los padres puede afectar a la educación y la salud de sus hijos y condicionar su salud, renta y bienestar futuros.

En este sentido, la educación es un factor clave para explicar los efectos a largo plazo de las crisis económicas sobre la población. La conexión causal entre educación y salud está bien establecida, y es bidireccional (Cutler et al., 2008, Groot y Maassen van den Brink, 2007, Groosman y Kaestner, 1997). Los beneficios de la educación sobre la salud constituyen uno de sus denominados «beneficios no monetarios». Los niños que gozan de buena salud tienen mejor rendimiento escolar y de adultos serán por ello más sanos, ya que acumulan habilidades cognitivas para procesar información sobre la producción de salud. Además, los niños más sanos son objeto de una inversión educativa más intensa por las expectativas de rendimiento a largo plazo y tendrán mejores oportunidades laborales por esta doble vía: mejor salud y más educación. Así, mejores oportunidades laborales se asocian a una mayor renta futura, lo cual también se asocia positivamente con la salud en edades medias y avanzadas de la vida. Por tanto, existe un círculo virtuoso entre buena salud en edad infantil y bienestar presente y futuro de la persona. Y, al contrario, padecer una mala salud puede derivar en un incremento considerable del riesgo de pobreza. Estos argumentos no son puramente teóricos. Existen pruebas empíricas bien fundamentadas de que la obligatoriedad de la educación hasta cierta edad en las legislaciones europeas ha tenido efectos beneficiosos sobre la salud (Cutler et al., 2008). Si, como efecto de la crisis, se pierden años de escolarización y aumenta el fracaso escolar, se

${ }^{1}$ Las crisis imponen restricciones presupuestarias exógenas a la sanidad y a las políticas sociales, lo que conlleva una reasignación de recursos. Según el Sistema de Cuentas de Salud, el gasto sanitario público no se ajustó inmediatamente tras la llegada de la crisis. De hecho, creció entre los años 2007 y 2010 desde 64.216 a 75.118 millones de euros. Llegado ese momento, los ajustes presupuestarios le hicieron caer hasta los 66.799 millones en el año 2014, para después volver a crecer hasta los 71.036 millones en el año 2015 (último año disponible en el momento de redactar estas líneas). 
acabará pagando la factura, a la larga, en términos de salud perdida y de un menor bienestar social.

Otros grupos que han de monitorizarse por ser potencialmente vulnerables son los ancianos, los desempleados y los inmigrantes. Cada uno de ellos está en riesgo por motivos diferentes que le son propios, pero tienen en común la posibilidad de desatención o pérdida de prioridad. La población mayor es la que acumula una mayor prevalencia de enfermedades crónicas, por lo que su estado de salud es más delicado y podría verse comprometido en mayor medida a consecuencia de cambios en sus condiciones de vida y determinantes de la salud, así como por la evolución de ciertas políticas públicas (pensiones, servicios sociales) afectadas por las crisis económicas. Por su parte, los desempleados están continuamente expuestos a sufrir de una manera más intensa los efectos de las crisis. El desempleo se ha asociado con un aumento de la mortalidad general, las muertes por suicidio y con diversos problemas de salud (Bambra et al., 2009; Brenner, 1979a; Brenner, 1979b; Brenner, 2005), aunque también se ha asociado empíricamente con la reducción de la mortalidad general y por causas específicas (enfermedades cardiovasculares, accidentes de tráfico y laborales), e incluso con mejoras de salud en distintos países y periodos de tiempo (Ruhm, 2000; Tom et al., 2007; Neumayer, 2004; Gerdtham y Ruhm, 2006, Jin et al., 1995). Esta disparidad de resultados es consecuencia, en parte, de las diferencias metodológicas encontradas en los estudios y, también en parte, de la realidad. Por un lado, el desempleo influye negativamente en la renta, de manera más o menos intensa dependiendo de la generosidad de las prestaciones económicas públicas del país (en cuantía y duración). El empobrecimiento derivado empeora la dieta, las condiciones de vida y la salud mental. Pero, por otra parte, el desempleo reduce el coste de oportunidad del tiempo y el estrés del trabajo, permite dormir más y dedicar tiempo al autocuidado de la salud. Además, reduce la exposición a accidentes laborales y de tráfico. A nivel contextual, el descenso de la producción industrial reduce la contaminación, afectando positivamente a la salud de la población residente en el área. Así pues, el efecto neto del desempleo sobre la salud dependerá de la resultante de esos vectores contrapuestos. El efecto de esas dos fuerzas de signo contrario marca el resultado final de la asociación entre desempleo y mortalidad, que varía de unas crisis a otras (Jin et al., 1995). En el apartado 2.3 se comparan resultados de estudios de diferentes años, con métodos estadísticos diferentes y resultados no coincidentes. Finalmente, los inmigrantes, aun cuando en el momento de llegada al país suelen disfrutar de un mejor estado de salud (efecto «inmigrante sano»), son más vulnerables a desarrollar peores estados de salud debido a su exposición a peores condiciones de vida y de trabajo, una situación laboral más precaria e incierta. Estos problemas son mucho mayores en el caso de inmigrantes en situación irregular, a lo que se les añaden los problemas de acceso a los servicios sanitarios, hecho que provoca un efecto mayor de las consecuencias económicas de la crisis sobre este subgrupo de población (Vázquez et al., 2014). 


\subsection{Problemas metodológicos: diseños y datos}

Como se deduce de lo expuesto, no es fácil cuantificar los efectos de la crisis económica sobre la salud. A los problemas generales de establecer con rigor los fenómenos de causación, se añaden otros problemas específicos relacionados con la cronología de la crisis y con las medidas de su intensidad. En los modelos causales, la causa es el fenómeno desencadenante y responsable de los efectos. Los estudios empíricos crisis/salud son de dos tipos: (i) aquellos para los que el tiempo es el proxy esencial de la crisis, donde se asocia determinados periodos temporales a las diferentes etapas pre-crisis, crisis, poscrisis, utilizando datos agregados; (ii) aquellos que cuantifican la causa (por ejemplo, porcentaje de desempleados, caída del PIB o del gasto público social) y estiman relaciones causa-efecto. Estos últimos pueden utilizar microdatos de individuos, familias, empresas, etc. o datos agregados para grupos de población, áreas geográficas o países.

Los estudios del primer tipo ajustan tendencias, estiman series temporales interrumpidas o segmentadas, realizan análisis jointpoint para detectar momentos de cambio de tendencia; en último término se basan en un diseño antes/después, atribuyendo en último término toda la variación del resultado (mortalidad, salud, utilización sanitaria) a la causa (crisis económica) cuyo proxy es el tiempo cronológico. Obviamente, ignoran las otras posibles causas coadyuvantes y contemporáneas a la crisis. Una dificultad añadida de los estudios donde el tiempo es el proxy esencial de la crisis económica es la decisión sobre la cronología de la crisis. Aunque los modelos econométricos de series temporales son capaces de estimar los retardos distribuidos en el tiempo como consecuencia de una intervención o causa instantánea en forma de impulso o escalón, la crisis económica no es un terremoto que ocurra en un instante, sino una secuencia continuada de malas noticias económicas que recorren la fase baja del ciclo. Es decir, no hay un antes y un después de la crisis económica (Librero et al., 2013). Por eso, algunos estudios han de hacer pruebas de robustez y análisis de sensibilidad para datar la propia crisis (Tapia Granados y Ionides, 2017). No obstante, en casos concretos, sí existe una fecha identificable para determinadas medidas reactivas a la crisis, como el cambio de los copagos de medicamentos, lo cual permite emplear con mayor garantía este tipo de modelos antes/después en situaciones concretas.

Los estudios del segundo tipo son más ambiciosos porque intentan asociar y atribuir efectos a causas. Pueden emplear datos temporales, transversales o de panel, con distinto nivel de agregación, desde microdatos hasta datos para países enteros. Los estudios empíricos de causa-efecto con series temporales agregadas se han utilizado ya desde antes de la reciente crisis para demostrar que las recesiones (mayores tasas de desempleo) no son necesariamente malas para la salud, pues la mortalidad puede reducirse durante la fase baja del ciclo económico (en el caso de EE. UU.) (Ruhm, 2000). Ese mismo autor, sin embargo, con datos más recientes (hasta 2010) y una metodología similar, concluye que ya las recesiones «no son lo que eran» y no es tan clara la relación procíclica con la mortalidad (Ruhm, 2015). Un paso más consiste en 
emplear datos longitudinales o paneles donde varios países, que se consideran comparables, se analizan a lo largo del tiempo, asociándose variables macroeconómicas explicativas con tasas de mortalidad por diversas causas, a la vez que se controla por variables demográficas y de contexto (Gerdtham y Ruhm, 2006).

Como hemos apuntado, los estudios difieren en los datos que emplean, tanto en el diseño como en la metodología estadística que aplican. La solidez de los resultados es muy variable. Los estudios observacionales, con datos transversales o cortes transversales repetidos, o bien con datos longitudinales (panel) tienen una limitación intrínseca de diseño, pero no es factible diseñar experimentos con la crisis como variable exógena de interés y solo en casos muy especiales se ha podido contar con datos cuasi experimentales en contexto de experimentos naturales. Los microdatos desagregados permiten estimar mejor las relaciones causa-efecto que los agregados, pues estos están sujetos al sesgo ecológico y, al fin y al cabo, las relaciones de causalidad (por ejemplo, entre desempleo y salud) se producen a nivel individual.

Aunque se están empleando datos de registros administrativos e historias clínicas individuales cada vez con mayor frecuencia, muchas veces la fuente se basa en muestras aleatorias representativas de grupos de población, que arrastran inevitables errores de muestreo. En muchos de los estudios, los datos proceden de encuestas, con los consiguientes problemas de valores perdidos no distribuidos aleatoriamente, sesgos de respuesta y de recuerdo, así como en subjetividad en medidas esenciales (peso, talla, comportamientos relacionados con la salud, etc.). Otro problema frecuente en las encuestas es la discontinuidad de algunas preguntas y el cambio de definiciones de una a otra edición. Además, hay sesgos claros en cuanto a la muestra empleada, puesto que habitualmente excluyen población institucionalizada, y dejan fuera a las «colas» de la población. Por ello, difícilmente podemos suponer que son encuestas representativas, por ejemplo, de la gente que vive en la calle, o de las personas que residen irregularmente en el país.

Por otra parte, una cosa es construir un modelo teórico sobre los mecanismos y procesos por los que las crisis económicas pueden acabar afectando a la salud, directa e indirectamente, a corto y a largo plazo, y otra tarea es cuantificar cada uno de los efectos implicados. Ello es extremadamente complejo y requiere métodos rigurosos, indicadores adecuados y distancia temporal (es decir, que haya transcurrido un tiempo suficiente para que los efectos de largo plazo se lleguen a apreciar). En cualquier caso, los efectos de la crisis económica sobre la salud tardan más en empezar a percibirse que los efectos sobre la renta y el bienestar económico de las familias y que los efectos sobre los servicios sanitarios.

\section{3. ¿Qué sabemos a partir de la experiencia de crisis anteriores?}

Las crisis anteriores en distintos países han sido analizadas en numerosas publicaciones y los resultados son sorprendentemente contradictorios, e incluso contraintuitivos en algunos casos (Márquez-Calderón, 2012). La idea general para explicar 
estas aparentes contradicciones es que no hay una «crisis económica estándar». El contexto importa y la intensidad y duración de la crisis también. Además, los resultados de los estudios están condicionados por las dificultades metodológicas que hemos expuesto en el apartado anterior.

Teniendo esto en cuenta, podemos señalar que en los países de rentas medias y bajas, las crisis económicas suelen tener mayor impacto porque afectan intensamente a los grupos de población más vulnerables. Los efectos se producen por el impacto simultáneo de la caída de la capacidad adquisitiva y de la pérdida de acceso a los servicios de salud, bien sea porque al perder el empleo se pierden también los derechos a la atención sanitaria, o bien porque los programas públicos de salud reducen su gasto. Así, por ejemplo, en México, la crisis de 1996 podría haber aumentado la mortalidad de niños (0-4 años) y de mayores de 60 años entre un 5 y un 7 por 100 . De hecho, se ha estimado que unos 7.000 niños y unos 20.000 mayores habrían muerto como consecuencia de aquella crisis (Cutler et al., 2000). Otro ejemplo es el caso de la crisis de 1997 de los «Dragones del Sudeste Asiático», la cual tuvo consecuencias trágicas para la salud de los grupos vulnerables, particularmente de los niños y de los más pobres (Tangcharoensathien et al., 2000; Waters et al., 2003). Indicadores como la incidencia de bajo peso al nacer y de los niños en edad escolar o la incidencia de malaria, sarampión y diarreas infantiles reflejan el efecto de la crisis, la cual se ha dejado notar incluso en las tasas ajustadas de mortalidad de Corea del Sur (Khang et al., 2005).

Un caso bien estudiado es el de Rusia. A raíz de la desintegración del régimen soviético y de la posterior puesta en marcha de estrategias de «terapia de choque» hacia el capitalismo, muchos de los países europeos del bloque oriental sufrieron un aumento considerable de la mortalidad. Entre 1990 y 1994, la mortalidad estandarizada por edad aumentó un 30 por 100 en Rusia y la esperanza de vida de los varones cayó de 64 a 58 años (la de las mujeres, de 74 a 71). Más del 75 por 100 de la caída de la esperanza de vida se debió a los aumentos de mortalidad de varones en edad laboral, sobre todo por causas externas y por enfermedad cardiovascular (Notzon et al., 1998; Walberg et al., 1998; Shkolnikov et al., 2001). Sin embargo, no es fácil establecer las causas, porque ocurrieron simultáneamente la desintegración institucional, la crisis económica, y los problemas de drogadicción, alcoholismo, malnutrición y deterioro del sistema de salud (Leon et al., 2007; Leon et al., 1997; Martin, 2002). La rápida privatización de las empresas, con el consiguiente aumento del desempleo, es una de las causas que se arguyen (Stuckler et al., 2009b), aunque esta justificación ya había sido objeto de intenso debate previo (Martin, 2002). Como contrapunto a Rusia, su vecina Finlandia sufrió una severa recesión en esos mismos años con un aumento del desempleo del 2 al 18 por 100, el cual, sin embargo, no tuvo efectos adversos sobre la salud, más bien al contrario. Durante la crisis disminuyó el consumo de alcohol, con efectos favorables sobre la mortalidad, disminuyeron los suicidios (Hintikka et al., 1999) y los intentos de suicidio (Ostamo y Lonnqvist, 2001) y se redujo el gradiente socioeconómico de la mortalidad (Valkonen et al., 2000). Las prestaciones del estado del bienestar en Finlandia amortiguaron los efectos de la crisis, mientras que la desintegración institucional en Rusia los agravó. 
Estados Unidos es el país donde más estudios se han realizado en este campo. Durante la Gran Depresión, entre 1929 y 1933, el producto interior bruto en EE. UU. cayó alrededor del 40 por 100 y el consumo per cápita un 30 por 100. El desempleo industrial subió del 7,9 al 26,1 por 100. Sin embargo, un efecto tangible a corto plazo fue el aumento de suicidios. La tasa de suicidios de varones blancos aumentó a inicios de los años treinta en los tramos de edad 45 y 74 años (MacMahon et al., 1963). La salud de los desempleados fue empeorando a lo largo de la recesión, no solo por el efecto renta, sino también por el sesgo de selección, a medida que los trabajadores de salud más frágiles iban siendo despedidos (Murray, 2007). Así, algunos estudios de la época (Sydenstricker, 1934) señalan que hubo mayor mortalidad en familias con sustentador principal sin empleo o trabajando a tiempo parcial, así como mayor incidencia de enfermedades en familias que experimentaron descensos en sus niveles de ingresos durante la recesión. Otros trabajos posteriores han continuado analizando la relación entre el cambio en el ciclo económico y la evolución de la salud de la población en este país, si bien los cambios en el ciclo han tenido intensidad y virulencia muy inferior en comparación con los casos presentados previamente. En los años setenta, varios estudios con datos temporales concluían que el desempleo tenía efectos negativos sobre la mortalidad (salvo por accidentes) y sobre la salud (Brenner, 1979a, Brenner, 1979b). Pero en los últimos años, aplicando métodos estadísticos de panel más sofisticados que controlan por efectos fijos del Estado (o la provincia) invariantes en el tiempo, diversos investigadores concluyen que se da la relación opuesta: la salud mejora en tiempos de crisis económica y empeora en años de bonanza. Deaton y Paxon (2001) concluyen que los aumentos de renta en épocas alcistas del ciclo hacen aumentar la mortalidad, por empeorar los estilos de vida. Por su parte, Ruhm (2005), basándose en un análisis con datos agregados para los diferentes estados de EE. UU. en los años 1972-1991, estima que las fases bajistas del ciclo se asocian a caídas de la mortalidad, y que un 1 por 100 de aumento del desempleo en los EE. UU. resulta en una reducción del 0,5 por 100 en la mortalidad general. No obstante, la subida del desempleo en esos años fue tan solo del 5,6 al 6,8 por 100, por lo que no puede hablarse de crisis, sino de cambio de ciclo económico. Otro estudio posterior del mismo autor con microdatos de adultos para 1987-2000 observa que cuando subía el desempleo en los EE. UU. disminuía ligeramente la mortalidad, al mismo tiempo que las personas reducían el consumo de tabaco y el exceso de peso, y aumentaban el ejercicio físico en el tiempo libre. Según las estimaciones de Ruhm (2005), la caída del 1 por 100 de la población ocupada reduce la prevalencia de tabaquismo, obesidad, inactividad física y múltiples riesgos para la salud en 0,6 por $100,0,4$ por $100,0,7$ por 100 y 1,1 por 100 , respectivamente. Esos efectos se han notado particularmente entre las personas con patrones de comportamiento más insanos: los grandes fumadores, los muy obesos y los que no hacían absolutamente nada de ejercicio físico. El trabajo más reciente de Strumpf et al. (2017) también muestra cómo las tasas de mortalidad en las áreas metropolitanas de EE. UU. se redujeron entre 2005 y 2010, mientras las tasas de desempleo aumentaban, y explica la mayor parte de ese efecto por la disminución de la mortalidad por 
enfermedades cardiovasculares. Por el contrario, constata un aumento de las muertes por sobredosis entre los adultos entre 25 y 54 años.

Otros estudios también han encontrado efectos procíclicos del desempleo sobre la salud en otros países de rentas altas, además de EE. UU. (Tapia Granados, 2005a): Francia (Tom et al., 2007), Alemania (Buchmueller et al., 2007) y para un grupo de 16 países de la OCDE (Gerdtham y Ruhm, 2006). También se han publicado resultados similares para España: Tapia Granados (2005b), con datos de las provincias españolas (1980-1997), en los que el desempleo osciló entre el 7 y el 24 por 100 y un modelo de panel de efectos fijos, concluye que la mortalidad aumenta procíclicamente cuando hay expansión económica y bajada del desempleo.

Así pues, la literatura científica de las décadas pasadas encontraba consistentemente que la mortalidad tiene comportamiento procíclico (Ruhm, 2000; Ruhm, 2005). Dicho brevemente, las crisis eran buenas para la salud. Sin embargo, los mismos autores han descubierto que ya no es así, o no tanto (para EE. UU.) (Ruhm, 2015). Además, señalan que los estudios con datos temporales necesitan series largas, de veinte años o más, para identificar relaciones de causa-efecto. La mortalidad cardiovascular y la derivada de los accidentes viales parece que sigue siendo procíclica, pero la mortalidad por cáncer repunta en tiempos de crisis, posiblemente porque el coste de tratar un caso de cáncer se ha multiplicado en la última década.

Por otra parte, aun cuando se observa que las crisis económicas no afectan a la evolución de las tasas de mortalidad o afectan en sentido favorable, cabe señalar que ello no implica que los resultados sean homogéneos en la población e incluso que la tendencia sea similar entre países. Blakely et al. (2008), durante la crisis económica que vivió Nueva Zelanda durante los años ochenta y noventa del pasado siglo, identificaron que la mortalidad cayó con más intensidad en la población de elevados ingresos en comparación con los más pobres. Por su parte, tomando como referencia el nivel educativo, Edwards (2008) identifica que, en EE. UU., en las recesiones experimentadas a lo largo del periodo 1979-1998, se produjo un incremento de la mortalidad de los varones en edad laboral de bajo nivel educativo, un descenso en los de elevado nivel educativo y se mantuvo estable en los de educación intermedia. En el caso de Noruega, durante el periodo 1977-2008, las fases bajistas del ciclo económico afectaron de manera más intensa a los grupos socioeconómicos más favorecidos en cuanto a educación o los ingresos, pero, cuando se clasificaba por riqueza, el grupo más afectado era el socioeconómicamente más desfavorecido (Tapia Granados e Ionides, 2017). En el caso de Finlandia, en la crisis ya reseñada de inicios de los años noventa, la mortalidad cayó en mayor proporción entre los trabajadores manuales que entre los no manuales (Valkonen et al., 2000). En Japón, en los mismos años, la mortalidad se incrementó entre profesionales de clase alta y directivos, pero no entre trabajadores manuales (Wada et al., 2012).

En suma, aunque tenemos buenos modelos teóricos que nos ayudan a entender los mecanismos de transmisión de la crisis, la intensidad de los efectos está mediada por la intensidad y duración de la propia crisis, así como por los elementos de contrapeso dotados desde la acción pública y comunitaria del país en cuestión. Por 
otra parte, no es sencillo cuantificar rigurosamente los efectos de la crisis económica sobre la salud. A los problemas generales de identificar relaciones de causa y efecto, a corto, medio y largo plazo, se añaden otros problemas específicos relacionados con la cronología de la crisis y las medidas de su intensidad, así como de la disponibilidad de datos en tiempo y forma adecuados para realizar los análisis.

Los resultados de los estudios realizados en distintos países sobre anteriores crisis son muy dispares. En muchos casos, las crisis se han visto acompañadas de mejoras en la salud poblacional, si bien en otras ocasiones se identifica el efecto contrario. Asimismo, se debe señalar que los efectos estimados no se distribuyen de manera homogénea entre la población del país analizado.

\section{Revisión de la literatura sobre España en la última década (2008-2018)}

En este epígrafe revisaremos la literatura sobre España publicada en revistas científicas acerca de las consecuencias de la reciente crisis en la salud de la población y en la persistencia o incremento de las desigualdades en salud. Para ello, nos apoyaremos en una reciente revisión realizada por dos de las autoras de este artículo (Gonzaléz López-Valcárcel y Barber, 2017), actualizándola al momento de la realización de este trabajo. La selección de artículos se centró en aquellos publicados entre 2008 y junio de 2018 en revistas con revisión por pares, en inglés o español.

\subsection{Mortalidad y causas de muerte}

Tapia Granados e Ionides (2017) analizan la evolución de la esperanza de vida al nacer y de la mortalidad para el periodo 1998-2014 en 27 países europeos, empleando tres bases de datos de la Organización Mundial de la Salud y el Banco Mundial y utilizando diferentes modelos de análisis de datos de panel. Sus resultados señalan que en aquellos países más afectados por la crisis (entre ellos, España) la reducción de la mortalidad fue más intensa en el periodo de crisis económica (2007-2010) que en la etapa anterior a la crisis (2004-2007). Los patrones de mortalidad variaron procíclicamente para el total, por sexo, por causas específicas de mortalidad (salvo suicidio, donde los resultados son débilmente contraclícicos) y en los tramos de edad 30-44 y 75 y más años.

El estudio realizado por Regidor et al. (2014) analiza la evolución de las tasas de mortalidad prematura para varias causas. Para cumplir su objetivo, los autores estudian la evolución de las tendencias temporales, comparando antes y después del inicio de la recesión (1995-2011). Con ello, consideran un total de 15 indicadores de salud: tasas de mortalidad prematura (general y específicas), y por causa de muerte, más dos indicadores específicos de morbilidad. Todos los indicadores considerados, con la excepción de la mortalidad por cáncer y la incidencia de VIH, mostraron una tendencia decreciente durante todo el periodo, incluyendo los años de recesión 
económica (2008-2011). Los autores apuntan que los resultados deben ser tomados con precaución, ya que los posibles efectos negativos de la crisis económica podrían manifestarse en el largo plazo, más que en el corto, y señalan la necesidad de estudios que continúen analizando los años posteriores a 2011.

Algunos estudios se centran en analizar causas seleccionadas de mortalidad, añadiendo matices al panorama general. Así, Ferrando et al. (2018) analizan las muertes por cáncer encontrando diferencias por tipo de cáncer y por sexo. Además, concluyen que la disminución de la tasa de mortalidad por cáncer se ha desacelerado en los años de crisis. Por su parte, Alonso et al. (2017) analizan la tendencia de mortalidad atribuible al alcohol. Sus resultados indican que durante los años de crisis se ha producido una importante heterogeneidad por subgrupos sociodemográficos, siendo especialmente desfavorable en las personas sin empleo. En cambio, Álvaro-Meca et al. (2018) identifican que las tasas de mortalidad por sepsis se incrementaron ligeramente en los años previos a la crisis, de la mano de un aumento de la incidencia, para estabilizarse en los años de crisis.

Por tanto, y en síntesis, los trabajos revisados no coinciden con ciertos mensajes catastrofistas que alertan de un exceso de medio millón de muertes en España, imputados a las políticas de austeridad, entre los años 2011 y 2015. Esos resultados estarían muy lejos de ser plausibles, pues se basan en datos mal estandarizados como se ha puesto de manifiesto por la literatura científica (Hernández-Quevedo et al., 2018).

Por otro lado, existen trabajos que asocian crisis económica y mayor desigualdad en mortalidad. Por ejemplo, un estudio reciente con datos de mortalidad en áreas pequeñas muestra cómo el riesgo de muerte en Barcelona ha aumentado mucho más en los barrios con mayor nivel de privación económica en comparación con los más ricos, lo que se traduce en un aumento de las desigualdades socioeconómicas en la mortalidad dentro de la ciudad entre 2009 y 2011 (Maynou et al., 2014). Por su parte, Ruiz-Ramos et al. (2014) constatan también un aumento en las desigualdades sociales en la mortalidad general de hombres entre 2007 y 2010, como consecuencia del mayor descenso en la mortalidad entre los individuos de mayor nivel de estudios en comparación con el resto, todo ello, en un contexto de descenso general de la mortalidad. Sin embargo, en un trabajo más reciente, Mackenbach et al. (2018) analizan las tendencias en resultados en salud de 27 países europeos y las comparan con los resultados de EE. UU., distinguiendo a la población por niveles educativos. Los resultados sugieren que el incremento de las desigualdades en salud observadas en EE. UU. no tiene un correlato similar en Europa. En el caso concreto de España, se señala que las tasas de mortalidad entre la población con nivel educativo más bajo y más alto han continuado disminuyendo rápidamente.

Los suicidios, por su parte, han sido uno de los problemas a los que los investigadores han dirigido mayores esfuerzos de análisis en estos años. De este modo, ha habido varios estudios empíricos sobre la dinámica de los suicidios e intentos de suicidio durante la crisis, sin que los resultados sean concluyentes (López-Bernal et al., 2013; Librero et al., 2013; Córdoba-Doña et al., 2014; Miret et al., 2014; Saurina et al., 2015). Concretamente, Laanani et al. (2015), utilizando datos de 
Eurostat, analizan el impacto de las variaciones del desempleo sobre las muertes por suicidio en ocho países de la Europa occidental entre los años 2000 y 2010 . Sus resultados indican que la tasa de suicidio se incrementa en un 0,3 por 100 para un aumento del 10 por 100 en la tasa de desempleo. No obstante, sus resultados son solo estadísticamente significativos para Holanda, Francia y Reino Unido. En España, no se encontró asociación entre desempleo y suicidio pese a ser el país con mayor tasa de paro. Un problema del estudio de Laanani et al. (2015) es que su serie temporal termina en 2010, cuando la crisis no había hecho más que comenzar. Los resultados y conclusiones no son muy diferentes en el trabajo de Fountoulakis et al. (2014), quienes emplean datos de Eurostat para 29 países europeos en el periodo 2000-2011. Para España, encuentran una correlación muy débil entre las tasas de suicidio y los indicadores macroeconómicos.

López-Bernal et al. (2013), realizan un análisis de series temporales para los años 2005-2010 analizando los datos mensuales de suicidio obtenidos de la Estadística de Defunciones por Causa de Muerte (EDCM) del Instituto Nacional de Estadística, desagregando por región y grupos de edad. Los autores estiman un incremento del 8 por 100 en la tasa de suicidio asociado a la crisis económica. No obstante, este estudio fue fuertemente respondido por Librero et al. (2013), poniendo en duda sus principales conclusiones, en especial, la identificación de una relación causa-efecto entre crisis económica y muertes por suicidios. Saurina et al. (2015), centrándose en Cataluña, y analizando el periodo 2000-2011 concluyen que el incremento observado en la tasa de suicidio no es estadísticamente significativo en conjunto aunque sí lo es para algunos grupos concretos: mujeres en edad laboral (16-64 años) residentes en municipios de 10.000 o más habitantes. Sin embargo, no se encontró asociación significativa entre el incremento de la tasa de desempleo y tasa de suicidio. Por su parte, Borrell et al. (2017) analizan los cambios de las muertes por suicidios en el País Vasco y la ciudad de Barcelona antes y durante la crisis económica, concluyendo que las tendencias en cuanto a desigualdad por esta causa de muerte por nivel educativo se mantuvieron relativamente estables. Por su parte, Rivera et al. (2017) analizan la tasa de suicidios por regiones españolas entre los años 2002 y 2013 empleando también la EDCM. Aplicando un modelo de datos de panel, encuentran que las tasas de suicidio se asocian significativamente con el desempleo, el desempleo de larga duración (de manera positiva) y la tasa de crecimiento del PIB (negativa). Empleando también la EDCM para el periodo 1980-2014, Álvarez-Gálvez et al. (2017) señalan que durante la primera parte de la crisis (2007-2011), la tendencia es decreciente aunque no estadísticamente significativa, mientras que en el segundo lapso temporal (2011-2014) se identifica una relación positiva y significativa entre el desempleo, el PIB per cápita y las tasas de suicidio. Por su parte, Ruiz-Pérez et al. (2017) apuntan un cambio de la tendencia en la reducción de la tasa de suicidio en los años 2008-2009 y 2012, especialmente en el caso de los varones y con una elevada heterogeneidad regional. Los escasos trabajos que manejan datos posteriores a 2011 les convierten en acreedores de especial interés al ampliar el periodo de estudio de años de crisis. No obstante, se ha de considerar que el Instituto Nacional de 
Estadística advierte en una nota sobre una mejora metodológica en el año 2013 que afecta a los casos de muerte afectados por procedimiento judicial (como es el caso de los suicidios). Sin embargo, los estudios no aclaran cómo han manejado este cambio.

Miret et al. (2014) estiman la prevalencia de ideación e intento de suicidio en población general en España comparando periodos anteriores a la crisis con años de crisis económica y analizando factores asociados para diferentes grupos etarios. Para ello, realizan una encuesta representativa de la población residente en España para los años 2011-2012 y comparan sus resultados con los del proyecto ESEMED, realizado en los años 2001-2002. La principal conclusión de los autores es que la prevalencia de la ideación e intentos de suicidio no cambió significativamente en el periodo objeto de estudio. Por su parte, Córdoba-Doña et al. (2014), centran su análisis en el impacto de la crisis sobre los intentos de suicidio registrados en Andalucía y en su relación con el desempleo, la edad y el sexo. Analizan un periodo temporal que cubre los cinco años previos a la crisis (2003-2007) y cinco años de crisis (20082012). Los autores encuentran un crecimiento de los intentos de suicidio en los años de crisis. Además, los adultos de 35 a 54 años fueron el colectivo más afectado. Asimismo, los intentos de suicidio se asocian a las tasas de desempleo en el caso de los varones mientras que este efecto no es significativo en el caso de las mujeres. Como principal limitación del trabajo se identifica que los intentos de suicidio se obtienen a través de las llamadas de emergencia al número 112, lo cual supone, por una parte, que los intentos no notificados no son observados ni, por tanto, analizados, $\mathrm{y}$, por otra, que cambios temporales en la utilización del número de emergencias son indistinguibles del efecto crisis.

En suma, la crisis, en términos generales no parece haber afectado en el corto plazo de manera negativa a las tasas de mortalidad. De hecho, algunos trabajos identifican para nuestro país, una relación procíclica entre las tasas de mortalidad y la evolución del PIB. Las desigualdades en materia de mortalidad podrían haber aumentado en los años de crisis, si bien las conclusiones no son totalmente coincidentes.

Con respecto a la literatura existente que analiza la relación entre la crisis económica y los suicidios, los resultados sugieren conclusiones diferentes, en ocasiones incluso contradictorias entre estudios. En todo caso, los efectos parecen ser diferentes en función del sexo y la edad. Además, la tasa de desempleo tampoco refleja una relación sólida y repetida con los suicidios. La mayoría de los estudios abogan por continuar con los análisis ya realizados extendiendo el lapso temporal para ayudar a clarificar la relación a largo plazo entre ciclo económico y causa de muerte.

\subsection{Salud autopercibida}

La salud autopercibida tampoco parece haber decaído durante la recesión (Regidor et al., 2014). De hecho, comparando las encuestas de salud de 2006, 2011 y 2014, se aprecia incluso una mejora en la percepción de la salud de la mayor parte de la población, a diferencia de lo que ocurre con otros países europeos donde la crisis 
económica fue menos dura (Bacigalupe et al., 2016). Esta mejora en la percepción de la salud podría asociarse a que en el ranking de prioridades durante los periodos de crisis económica, la salud pierde puestos con respecto a otras preocupaciones (como el trabajo o las dificultades económicas).

Arroyo et al. (2015), empleando las Encuestas Nacionales de Salud (ENS) de 2006 y 2011 y sumando las Encuestas de Salud de Cataluña (ESCA) de 2006 y 2011, no encuentran un empeoramiento en la salud autopercibida en los años de crisis de la población general. Aguilar-Palacio et al. (2015) utilizan la ENS (ediciones de 2001 a 2011-12) y la Encuesta Europea de Salud de 2009 para analizar la evolución de la relación entre el nivel educativo y la salud percibida. Las autoras concluyen que la prevalencia de mala salud percibida fue mayor en las mujeres con nivel educativo bajo y mejoró en las de nivel educativo alto, observándose importantes diferencias entre CCAA. Además, aunque la prevalencia de mala salud percibida no varía en el periodo analizado, se identifican desigualdades en su evolución según el nivel educativo y el sexo.

Utilizando las Encuestas de Condiciones de Vida (ECV) de 2007 y 2011, Calzón et al. (2017) muestran que la buena salud percibida entre la población se incrementó del 75 por 100 en 2007 a un 83 por 100 en 2011. La menor probabilidad de presentar mala salud percibida fue estadísticamente significativa tanto en varones como en mujeres. Además, un factor de riesgo para la mala salud percibida en los varones fue el desempleo, mientras que para el caso de las mujeres no hubo significación estadística alguna. Empleando las mismas encuestas, Fornell et al. (2018) identifican una mayor probabilidad de reportar mala salud percibida en aquellas personas desempleadas (odds ratio u $\mathrm{OR}^{1}$ de 1,78 respecto a las empleadas), con inseguridad laboral (OR de 1,38 respecto a las que no indicaban inseguridad) o que formaban parte de un hogar con privación material (OR de 1,87 respecto a los hogares sin privación). También utilizando las ECV, López del Amo et al. (2018) apuntan resultados similares. En este sentido, el trabajo previo de Urbanos-Garrido y González López-Valcárcel (2013) también mostraba una asociación positiva y significativa entre desempleo y mala salud percibida, particularmente intensa cuando el desempleo es de larga duración.

Por otra parte, el impacto de la crisis en las desigualdades sociales en salud autoreportada difiere en función de qué indicador se emplee para medir la situación socioeconómica de los individuos. Así, Coveney et al. (2016) muestran que la crisis tuvo como resultado un brusco descenso de la desigualdad en salud relacionada con la renta entre 2009 y 2012 (medida mediante índices de concentración), que se deriva de un efecto perverso: el desempleo y la caída de ingresos para los empleados jóvenes desplazaron a los grupos de población más sanos a la parte baja del ranking de rentas, mientras que los pensionistas (con peor salud) mantuvieron su renta nominal a salvo. Sin embargo, Barroso et al. (2016) comprueban que, aunque las

${ }^{1}$ El odds ratio (conocido por sus siglas OR) es el cociente entre la probabilidad de que ocurra un evento y la probabilidad de que no ocurra. 
desigualdades sociales en la salud autopercibida relacionadas con el nivel educativo parecen aumentar entre 2006 y 2011-2012, las relacionadas con la situación profesional de los propios individuos y de los sustentadores principales del hogar parecen verse atenuadas.

\subsection{Salud mental}

El impacto de la crisis sobre la salud mental está bien documentado (Frasquilho et al., 2016). En general, los estudios apuntan a que la crisis se asocia con un crecimiento en la prevalencia de los trastornos mentales y a un incremento del riesgo a padecer problemas de este tipo. Sin embargo, este empeoramiento ha sido desigual. Varios estudios identifican que el agravamiento de los problemas mentales se ha dado sobre todo en varones en edad laboral, especialmente en aquellas personas en situación de desempleo o con empleos precarios, y también se ha asociado con la presencia de problemas financieros en el hogar. Ello sugiere que el canal de causalidad se da entre mercado laboral-desempleo/precarización y enfermedad mental y que, por tanto, son las políticas sociales, más que las sanitarias, las que potencialmente pueden mitigar y revertir esta situación.

Gili et al. (2013) analizan en su estudio los contactos de la población con los servicios sanitarios de atención primaria (AP) durante los años 2006-07 y los comparan con los años 2010-2011. La principal conclusión es que hubo un fuerte incremento de los trastornos mentales y del abuso de alcohol entre la población que utilizó los servicios de AP, especialmente entre las personas que padecían desempleo y sus familiares y en aquellas personas con problemas para pagar su vivienda. Bartoll et al. (2014) analizan cambios en la salud mental en España y en la desigualdad en su prevalencia entre 2006 y 2011-2012 empleando las Encuestas Nacionales de Salud (ENS). Los autores identifican un incremento de la prevalencia de mala salud mental en varones, atribuido al estatus laboral, y un ligero descenso en mujeres. Asimismo, se identifica un aumento de las desigualdades socioeconómicas en salud mental en el caso de los hombres, mientras que en las mujeres la desigualdad parece no registrar cambios apreciables. Urbanos-Garrido y González López-Valcárcel (2013) estiman el impacto del desempleo (especialmente el de larga duración) sobre la salud mental de la población en edad activa y evalúan si durante la crisis dicho efecto se ha intensificado. Empleando también las ENS de los años 2006 y 2011-2012, las autoras apuntan que el desempleo tiene un impacto negativo y estadísticamente significativo sobre la salud mental. Este impacto se amplifica en el caso del desempleo de larga duración, siendo significativamente más intenso durante la crisis económica. Por su parte, Gili et al. (2016) analizan datos de 7.914 pacientes encuestados en los años 2006-2007 y de 5.876 en los años 2010-2011, a través de la entrevista Primary Care Evaluation of Mental Disorders e identifican un aumento de prevalencia del Trastorno Depresivo Mayor (incremento del 156 por 100 en varones y del 105 por 100 en mujeres), y del Trastorno de Ansiedad Generalizada (aumento del 98 por 100 en 
varones y del 71 por 100 en mujeres). La variable «estar desempleado» fue estadísticamente significativa en todos los análisis.

Basterra (2017), emplean también datos de las ENS para evaluar la prevalencia de alto riesgo de morbilidad psiquiátrica en población adulta española. Los principales resultados apuntan a que la prevalencia de alto riesgo de morbilidad psiquiátrica apenas se movió entre ambas ediciones (20,5 por 100 en 2012 vs. 21,3 por 100 en 2006). Diferenciando por sexo, la prevalencia descendió en mujeres y se incrementó en varones en edad laboral. Ruiz-Pérez et al. (2017) analizan el papel de factores contextuales asociados a problemas de salud mental durante la recesión. Empleando las ENS, sus resultados muestran un empeoramiento de la salud mental a lo largo de la crisis, e indican que las principales variables macroeconómicas asociadas a dicho empeoramiento fueron el gasto sanitario per cápita y el porcentaje de empleo temporal (como variable proxy de la precarización laboral). Medel Herrero et al. (2017) examinan la relación entre la crisis económica y el número de hospitalizaciones psiquiátricas. Los autores señalan que las tasas de altas hospitalarias psiquiátricas ajustadas por edad aumentaron significativamente a partir de abril del 2008, coincidiendo con el inicio de la crisis, especialmente en pacientes de entre 15 a 24 años de edad y, en menor medida, entre los 25 a 34. No se observó efecto en el resto de tramos de edad.

Otros trabajos destacables emplean bases de datos locales o regionales. IglesiasGarcía et al. (2014) concluyen que, para el caso de Asturias, las variaciones de los indicadores económicos seleccionados durante el periodo de crisis (hasta 2010) no se asocian con un incremento en la demanda asistencial por problemas mentales, si bien existe una correlación negativa entre la tasa de paro y la demanda asistencial. En el caso de Andalucía, la recesión económica se asocia a peor salud mental, con diferencias marcadas según el estatus laboral y el nivel educativo, asociándose los problemas financieros y el apoyo social de manera significativa con la salud mental en todos los subgrupos estudiados (Córdoba-Doña et al., 2016). En el País Vasco, Bacigalupe et al. (2016) muestran un claro deterioro de la salud mental desde el año 2008, especialmente en varones. Para este grupo, el deterioro afectó a todas las categorías laborales analizadas (exceptos los jubilados), y entre las mujeres afectó a las desempleadas. En el área de Badalona, Sicras-Mainar y Navarro-Artieda (2016) identifican un mayor uso de medicamentos antidepresivos, lo cual asocian a un crecimiento de la prevalencia de depresión mayor, que pasó del 5,4 por 100 en el periodo 2008-2009 al 8,1 por 100 en 2012-2013. Asimismo, Barceló et al. (2016), utilizando también datos de Cataluña, confirman el incremento del consumo de medicamentos psicotrópicos respecto a los años previos a la crisis, alcanzándose el pico de consumo en el año 2011 y siendo más probable el mismo en la población desempleada.

En relación con trabajos cuyo objeto es el análisis de las desigualdades en salud mental, algunos trastornos como la depresión mayor parecen tener un gradiente social claro que podría haber aumentado durante la crisis, al menos en el caso de los varones (Bartoll et al., 2014). Moncho et al. (2018) empleando también las ENS de 
2006 y 2011-2012 apuntan que la prevalencia de población en riesgo de mala salud mental (empleando el General Health Questionnaire -GHQ - de Goldberg) se había incrementado en los varones y reducido en las mujeres. Sin embargo, los propios autores reconocen la complejidad de interpretar los efectos identificados, toda vez que el empeoramiento en los factores socioeconómicos relacionados con la crisis se asocia a un aumento de la prevalencia de riesgo de mala salud mental, pero también identifican una reducción en la prevalencia de ciertas enfermedades somáticas (cardiovascualres y osteoarticulares) durante los años de crisis, más marcada en los varones desempleados. Asimismo, en el caso de las mujeres, la reducción en la prevalencia del riesgo de mala salud mental no se asocia ni con cambios en factores socioeconómicos ni con cambios en las enfermedades somáticas. Reibling et al. (2017) emplean la European Social Survey para analizar la salud mental de la población de 21 países europeos durante el periodo 2006-2014, empleando la escala del Center of Epidemiological Studies-Depression (CES-D) para identificar sentimientos de depresión. Los autores señalan que las personas que declararon sentirse deprimidas se habían reducido entre 2006 y 2014 en todos los países, salvo en Chipre y en España. Las desigualdades previas se mantuvieron durante la crisis pero, en términos generales, no aumentaron. De hecho, en los cuatro países con el mayor aumento en sus tasas de desempleo (Portugal, Irlanda, Chipre y España), las desigualdades parecen haber disminuido entre 2006 y 2012, para mantenerse estables o aumentar de nuevo hasta el final del periodo.

En suma, hay evidencia consistente entre estudios en el sentido de que la salud mental empeora durante la crisis, particularmente entre los varones, que ese empeoramiento podría asociarse al desempleo y al deterioro de las condiciones laborales, y de que las desigualdades sociales en salud mental se han mantenido durante los años de crisis o incluso podrían haber aumentado.

\subsection{Problemas de salud en grupos seleccionados}

Uno de los grupos vulnerables de población donde los efectos de las crisis pueden ser inmediatos y tener consecuencias a largo plazo son los niños (Rajmil et al., 2015). En Cataluña, durante los años de crisis, ha aumentado el gradiente social en la obesidad infantil y en las desigualdades en calidad de vida relacionada con la salud (Rajmil et al., 2013). Las consecuencias a largo plazo de las privaciones de los niños de hoy serán previsiblemente importantes en el futuro (Flores et al., 2014), de ahí que se abogue por la intervención pública temprana desde sectores como la educación para garantizar una nutrición adecuada. Arroyo-Borrell et al. (2017), utilizan la ENS para analizar si el estado de salud de las madres y sus determinantes socioeconómicos pueden influir en la salud mental de los hijos. Entre los principales resultados mostrados por las autoras cabe destacar la identificación de que el riesgo del niño a padecer un desorden mental se incrementa con los problemas mentales de la madre. Asimismo, el presentar un bajo estatus socioeconómico se asocia con un incremento 
del riesgo del niño a padecer problemas de comportamiento. En cambio, un elevado nivel educativo de la madre sería un factor protector frente al riesgo a padecer estos problemas. Los resultados apuntan a que la crisis económica no ha cambiado, en general, los trastornos mentales de los niños, si bien el riesgo de padecer problemas mentales es mayor en niños cuyos padres se encuentra en situación de desempleo.

Por su parte, en relación a la población mayor, (Benmarhnia et al., 2014) sugieren que del análisis de las series mensuales de mortalidad por edad y sexo de personas de 60 y más años desde 1995 a finales de 2012, la mortalidad parece descender a una velocidad inferior a la previsible ante la ausencia de la crisis, siendo mayor el posible impacto en mujeres que en varones. Sin embargo, con una serie temporal más larga (36 años, 1981-2016) y un análisis metodológicamente riguroso de la mortalidad por tramos quinquenales de edad, desde los 60 años, utilizando regresión segmentada (Joint-Point regression), Márquez et al. (2018) encuentran que la tendencia decreciente de la mortalidad se ha estancado o ralentizado en varones de 60 a 69 años, en menor medida en hombres de 70 a 79 y no se aprecian cambios de tendencia notables en edades avanzadas (80 años y más). En mujeres, hay cierto estancamiento en los años de crisis, que según la edad empieza ya en 2009 o en 2013, y solamente para las de edades entre 75 y 79 años la tendencia de la mortalidad se ha ralentizado desde 2010. Los autores señalan que estos hechos se han producido también en otros países del entorno como Inglaterra y Gales y que los intervalos de confianza de las estimaciones son amplios. Asimismo, los autores son extremadamente prudentes a la hora de atribuir esos hechos hallados a causas relacionadas con la situación económica o las políticas públicas durante los años analizados.

La obesidad es un problema de salud y un problema social de relevancia en España, donde según la ENS de 2017, que sesga a la baja la prevalencia al ser datos autodeclarados, se estima en adultos en el 16,7 por 100 de las mujeres y en el 18,2 por 100 de los hombres. La obesidad infantil (2 a 17 años) en 2017 se sitúa en el entorno del 10,3 por 100 de niños y niñas obesos. Considerando la dimensión territorial, hay diferencias muy marcadas: la prevalencia de obesidad en adultos es del 9,6 por 100 en Castilla León y del 21,4 por 100 en Canarias; la obesidad infantil es del 1,4 por 100 en Navarra y del 14,4 por 100 en Andalucía. Estas diferencias territoriales resultan en parte del gradiente social, que se superpone al territorial y es particularmente intenso para las mujeres. Así, en 2017, el 8,5 por 100 de las mujeres entre 25 y 64 años con estudios superiores eran obesas, mientras que la prevalencia era del 21,6 por 100 para las de nivel básico de estudios o inferior. El gradiente social en la obesidad se ha mantenido o aumentado entre 2006 y 2014 (Rodríguez-Caro et al., 2016), siendo la educación un factor determinante más influyente que la renta o la ocupación. Tanto la gravedad del problema como su gradiente social son mayores para las mujeres que para los varones. De hecho, la obesidad no es solamente un problema de salud que impone un coste social elevado, también conlleva problemas extrasanitarios como la discriminación laboral que sufren en España las mujeres con obesidad mórbida en empleos de cara al público y en comunidades autónomas con baja prevalencia de obesidad poblacional (Vallejo-Torres et al., 2018). 
La crisis económica sirvió de acicate para alinear los incentivos de los departamentos de Hacienda y Sanidad. Las propuestas de crear un impuesto sobre las bebidas azucaradas, responsables en gran medida de la obesidad infantil y juvenil (Ortún et al., 2016) han cristalizado de momento en una comunidad autónoma, Cataluña, con un impuesto específico en tres tramos en vigor desde mediados de 2017 y, sobre todo, en llevar a la agenda política la conveniencia del impuesto en las demás comunidades autónomas y en el Ministerio de Sanidad.

Por su parte, empleando las ENS desde 2003 hasta 2011, Zapata Moya et al., (2015) sugieren que «los efectos de la crisis sobre la salud surgen especialmente en el caso de nuestras enfermedades más prevenibles y entre los grupos de educación más baja». Ese gradiente educativo se encontraría presente en episodios de infarto de miocardio, diabetes mellitus, depresión y cáncer. Otro estudio que emplea las ENS concluye que las desigualdades socioeconómicas en la salud y en los comportamientos saludables se han intensificado en el periodo 2011/12, especialmente respecto a la dieta seguida (Bartoll et al., 2015).

La población en situación de desempleo también es un grupo potencialmente vulnerable ante la crisis económica. Como ya se ha señalado, toda vez que estos estudios se han centrado en suicidios (consumación e ideación), salud autopercibida y trastornos mentales, nos remitimos a los resultados expuestos sobre personas desempleadas en los anteriores apartados.

Por otra parte, Agudelo-Suárez et al. (2013) estudian los efectos de la crisis económica en España sobre la salud mental de inmigrantes trabajadores. Sobre una muestra de 318 personas, se identificó la presencia de mala salud mental empleando técnicas de entrevista personal (año 2008) y telefónica (2011). Los autores concluyen que la salud mental de las personas encuestadas había empeorado durante los años de crisis. Los subgrupos más afectados eran las personas desempleadas o con bajos salarios y con cargas familiares. Gotsens et al. (2015) analizan las desigualdades en salud entre la población inmigrante nacida en países de rentas medias y bajas y los españoles nativos, empleando las ENS de los años 2006 y 2011-2012. Los resultados indican que las desigualdades en salud percibida son mayores entre mujeres inmigrantes $v s$. nativas, mientras que en el caso de los varones las desigualdades se identifican en mala salud mental. Las autoras concluyen que entre 2006 y 2012 la salud de las personas inmigrantes que llegaron a España antes del año 2006 empeoró en comparación con la población nativa, quedando eliminado el llamado «efecto del inmigrante sano» en un contexto de crisis económica profunda. Sin embargo, Henares-Montiel et al. (2018) analizan desigualdades entre población inmigrante y nativa en España empleando la oleada española de la Encuesta de Salud Europea del año 2014. Los resultados indican que la población inmigrante presenta menos problemas físicos y mentales que los nativos, así como un menor consumo de psicofármacos. Las autoras concluyen que, transcurridos los años más duros de crisis económica, parece recuperarse el efecto «inmigrante sano», si bien las diferencias en salud observadas entre varones y mujeres nativos se reproducen en la población inmigrante. 


\section{Conclusiones y recomendaciones}

La revisión de la literatura sobre los efectos de la reciente crisis en España no ha identificado un impacto negativo sobre la evolución de la mortalidad, en general, si bien existe polémica sobre la evolución de la tasa de suicidios. Además, parece que la mortalidad de las personas mayores, entre 60 y 79 años, sobre todo de los varones, ha ralentizado su buena tendencia durante los años de crisis. En materia de salud autopercibida tampoco se detecta un empeoramiento general, si bien sí se identifican grupos cuya evolución ha sido negativa (desempleados, especialmente, los de larga duración y mujeres sin estudios). Los trabajos que analizan los problemas de salud mental identifican un impacto negativo de la crisis en esta dimensión, más intenso en varones, siendo especialmente relevantes el estatus laboral y el nivel de estudios. Por grupos de población, las cifras y el gradiente social en materia de obesidad infantil son preocupantes. En el caso de los inmigrantes, su salud podría haber empeorado en los primeros años de crisis para recuperarse al final de la misma. No obstante, este resultado hay que tomarlo con precaución debido a que los análisis se han realizado con datos de corte transversal y, por tanto, no analizan a las mismas personas durante un periodo continuado de tiempo ni controlan por las salidas ni llegadas de nuevos inmigrantes.

Finalmente, no está claro que, en el corto plazo, las desigualdades en salud de la población se hayan intensificado debido a la crisis: los resultados y conclusiones difieren por la diversidad de los periodos utilizados en los análisis, la heterogeneidad de las variables socioeconómicas y de salud consideradas, los cambios en el perfil socioeconómico de los grupos en comparación con los periodos previos y durante la crisis y el tipo de medidas utilizadas para analizar la magnitud de las desigualdades sociales en salud (Bacigalupe y Escolar-Pujolar, 2014).

Así pues, la principal conclusión que extraemos es que la crisis, pese al sufrimiento y a la pérdida de bienestar causados, no parece haber afectado de una manera severa, al menos en el corto plazo, a la salud de los españoles ni a la de los extranjeros residentes en España, salvo en la dimensión de salud mental. No obstante, cabe matizar que los resultados observados para la población general no deben ocultar las grandes diferencias identificadas entre grupos sociales y por estatus de ocupación. Asimismo, estos resultados se ciñen al corto plazo. Como señalamos en el apartado dos, la pérdida de empleo y de la caída de las rentas familiares, y el empobrecimiento que ello conlleva, supone uno de los mecanismos transmisores de las crisis sobre la salud. En esta materia, los indicadores socioeconómicos tales como las tasas de desempleo, indicadores de desigualdades de renta o las tasas de pobreza y de riesgo de exclusión social han mostrado un deterioro alarmante en estos últimos años, que es todavía más preocupante porque ya partían de un nivel elevado durante los años previos a la crisis económica. Ello alerta sobre efectos de la crisis en la salud que podrían manifestarse en el futuro, transcurrido cierto tiempo, lo cual obliga a ser cautelosos a la hora de extrapolar las conclusiones derivadas de los indicadores de salud actuales al medio-largo plazo. 
Algunas recomendaciones sobre actuaciones concretas en diversos ámbitos de las políticas públicas serían las siguientes:

1. Dado que los factores de riesgo identificados tienen su origen fuera del ámbito sanitario, aunque el sistema sanitario debe estar preparado para atender las mayores necesidades en salud que pueden surgir en momentos de crisis y posteriores, una buena parte de las políticas en salud deben desarrollarse fuera del medio sanitario.

2. La escuela es un lugar idóneo para mejorar la salud y el bienestar de los hoy niños y mañana adultos. La pobreza infantil se asocia a un mayor fracaso escolar y, por tanto, a menores oportunidades futuras y peor salud y bienestar. Las políticas educativas deben ser prioritarias (aún más) en años de crisis y los recursos destinados a alumnos con problemas escolares o necesidades especiales no deben ser los primeros en sufrir los ajustes presupuestarios, sino al contrario.

3. La escuela es también un lugar donde educar en salud. Los programas efectivos de educación alimentaria y de educación en comportamientos saludables (actividad física, prevención de acoso y maltrato, consumo de alcohol, tabaquismo, drogas ilegales, conductas sexuales de riesgo) deben tener en la escuela un ámbito prioritario de actuación.

4. Posiblemente las políticas que más han contribuido a evitar el deterioro de la salud y el bienestar de la población han sido políticas extrasanitarias como son el caso de la prolongación de las rentas recibidas por personas en paro una vez agotado el subsidio por desempleo, así como el mantenimiento de las pensiones. De hecho, los escenarios futuros sobre la evolución del sistema de pensiones no son precisamente tranquilizadores, lo que previene contra la tentación de dar por hecho que en un futuro los indicadores de salud de la población de más edad vayan a estar protegidos por una renta permanente y suficiente. Sin estas políticas, junto con el papel clave de otros pilares básicos del Estado del Bienestar como son el sistema sanitario y el educativo, la crisis económica hubiera derivado en una crisis social de mucha mayor magnitud. En relación con futuras crisis, el mantenimiento de una red última de garantía de rentas para todas las personas en situación de vulnerabilidad (jóvenes, mayores, desempleados) debe ser una política social de la mayor prioridad.

5. Dado que el ámbito laboral se ha revelado como un marco donde pueden estar aumentando o cronificándose riesgos para la salud, el desarrollo de políticas de salud laboral debería impulsarse con mayor firmeza. Asimismo, debe considerarse que las personas desempleadas (especialmente las de larga duración) son un grupo de población especialmente vulnerable, por lo que han de diseñarse políticas específicas dirigidas a este colectivo.

6. Otras políticas que han contribuido a mantener los indicadores de salud son las concernientes al consumo de tabaco, así como las de seguridad vial, que han logrado una gran reducción de muertes prematuras. En este sentido, aun 
felicitándose por los resultados, conviene sugerir que se debe perseverar para evitar una relajación en el cumplimiento de las normas que se encuentran asociadas a la favorable evolución de los resultados en salud en estos ámbitos.

7. Dentro del ámbito sanitario, hemos visto que la prevalencia de trastornos mentales en ciertos colectivos es notablemente superior a la media. Una crisis económica puede incrementar la necesidad de servicios sanitarios especializados en esta área. Dado que el punto de entrada en el sistema y el lugar donde se diagnostica tempranamente (idealmente) es la atención primaria, deberían ponerse en marcha programas especialmente dirigidos a reforzar los servicios sanitarios en las especialidades de atención primaria y psiquiatría, orientados especialmente a los grupos de población más vulnerables.

8. Las desigualdades sociales en salud siguen estando presentes. Por ello se recomienda diseñar una estrategia que permita poner en práctica de manera ordenada políticas e intervenciones para reducir las desigualdades sociales existentes en salud dentro de nuestro país.

Aunque este artículo se centra en analizar los resultados en salud y, por ello, no hemos entrado en el análisis de cómo la crisis ha afectado al sistema sanitario público, es evidente que siempre, pero en momentos de crisis especialmente, el uso eficiente de los recursos debería convertirse en una norma que orientara la asignación de los mismos. Aunque no profundizamos en este ámbito, señalaremos que las propuestas recogidas en el documento de debate de la Asociación de Economía de la Salud «La sanidad pública ante la crisis. Recomendaciones para una actuación pública sensata y responsable» y las 166 propuestas planteadas por la misma asociación en materia de reforma del Sistema Nacional de Salud siguen plenamente vigentes (AES, 2008; AES, 2013).

Los anteriores puntos deben operar bajo la premisa de que los programas, actuaciones, estrategias y políticas implementados deben ser evaluados de manera sistemática. El menú de intervenciones es prácticamente ilimitado. Sin conocer los recursos empleados y el retorno alcanzado (en términos de salud, de beneficios sociales, de bienestar) la asignación de los recursos (siempre escasos, pero más en momentos como los analizados) no podrá ser ni eficiente ni equitativa. Así, la evaluación de las políticas y la rendición de cuentas a la ciudadanía, como principios fundamentales de Buen Gobierno de la sanidad pública, deben estar presentes desde las fases de diseño (evaluación ex ante), implementación (evaluación durante) y tras la finalización de una fase o del despliegue completo de la política (evaluación ex post).

Al tiempo, no se debe dejar de investigar sobre los efectos de la crisis en la salud de la población. Hasta el momento no hemos hecho sino observar el corto plazo. Pero las cicatrices que ha dejado esta crisis son profundas. Está por ver si serán transitorias, duraderas o incluso permanentes en amplias capas de la población. De la crisis económica reciente se pueden aprender valiosas lecciones para evitar dolor y sufrimiento en las venideras. Porque, mientras no se demuestre lo contrario, el sistema económico vigente seguirá experimentando ciclos. Por tanto, la cuestión no es 
si volveremos a vivir crisis económicas, sino cuándo se producirán, qué intensidad tendrán, a través de qué canales se trasladarán a la población, cuáles serán los grupos más afectados y cuál será nuestra respuesta colectiva para contrarrestar sus efectos más negativos.

\section{Referencias bibliográficas}

[1] AGUDELO-SUÁREZ, A.; RONDA, E.; VÁZQUEZ-NAVARRETE, M. L.; GARCÍA, A. M.; MARTÍNEZ, J. M. y BENAVIDES, F. G. (2013). «Impact of economic crisis on mental health of migrant workers: what happened with migrants who came to Spain to work?». International Journal of Public Health, 58 (4), 627-631.

[2] AGUILAR-PALACIO, I.; CARRERA-LASFUENTES, P. y RABANEQUE, M. (2015). «Salud percibida y nivel educativo en España: tendencias por comunidades autónomas y sexo (2001-2012)». Gaceta Sanitaria, 29 (1), 37-43.

[3] ALONSO, I.; VALLEJO, F.; REGIDOR, E.; BELZA, M. J.; SORDO, L.; OTEROGARCÍA, L. y BARRIO, G. (2017). «Changes in directly alcohol-attributable mortality during the great recession by employment status in Spain: a population cohort of 22 million people». J. Epidemiol. Community Health, jech-2016-208759.

[4] ÁlVAREZ-GÁlVEZ, J.; SALINAS-PÉREZ, J. A.; RODERO-COSANO, M. L. y SALVADOR-CARULLA, L. (2017). «Methodological barriers to studying the association between the economic crisis and suicide in Spain». BMC Public Health, 17 (1), 694.

[5] ÁLVARO-MECA, A.; JIMÉNEZ-SOUSA, M. A.; MICHELOUD, D.; SÁNCHEZLÓPEZ, A.; HEREDIA-RODRÍGUEZ, M.; TAMAYO, E. y RESINO, S. (2018). «Epidemiological trends of sepsis in the twenty-first century (2000-2013): an analysis of incidence, mortality, and associated costs in Spain». Population Health Metrics, 16 (1), 4 .

[6] ARROYO, E.; RENART, G. y SÁEZ, M. (2015). «How the economic recession has changed the likelihood of reporting poor self-rated health in Spain».Int.J. Equity Health, $14,149$.

[7] ARROYO-BORRELL, E.; RENART, G.; SAURINA, C. y SÁEZ, M. (2017). «Influence maternal background has on children's mental health». International Journal for Equity in Health, 16 (63), 63.

[8] ASOCIACIÓN DE ECONOMÍA DE LA SALUD (AES). (2008). «Aportaciones al diagnóstico sobre el SNS español para un Pacto por la Sanidad». Barcelona: Asociación de Economía de la Salud. En http://www.aes.es/Publicaciones/sintesispactoMSC.pdf.

[9] ASOCIACIÓN ECONOMÍA DE LA SALUD (AES). (2013). «Sistema Nacional de Salud: diagnóstico y propuestas de avance». Barcelona, Asociación de Economía de la Salud.En http://www.aes.es/Publicaciones/sns2013_Resumen_documento.pdf.

[10] BACIGALUPE, A. y ESCOLAR-PUJOLAR, A. (2014). «The impact of economic crises on social inequalities in health: what do we know so far». Int. J. Equity Health, 13 (1), 52 .

[11] BACIGALUPE, A.; ESNAOLA, S. y MARTÍN, U. (2016). «The impact of the Great Recession on mental health and its inequalities: the case of a Southern European region, 1997-2013». International Journal for Equity in Health, 15 (1), 1475-9276. 
[12] BAMBRA, C.; POPE, D.; SWAMI, V.; STANISTREET, D.; ROSKAM, A.; KUNST, A. y SCOTT-SAMUEL, A. (2009). "Gender, health inequalities and welfare state regimes: a cross-national study of 13 European countries». Journal of Epidemiology and Community Health, jech-2007.

[13] BARCELÓ, M. A.; COLL-NEGRE, M.; COLL DE TUERO, G. y SÁEZ, M. (2016). «Effects of the Financial Crisis on Psychotropic Drug Consumption in a Cohort from a Semi-Urban Region in Catalonia, Spain». PloS one, 11 (2), e0148594.

[14] BARROSO, C.; ABÁSOLO, I. y CÁCERES, J. J. (2016). «Health inequalities by socioeconomic characteristics in Spain: the economic crisis effect». International Journal for Equity in Health, 15 (1), 62.

[15] BARTOLL, X.; TOFFOLUTTI, V.; MALMUSI, D.; PALÈNCIA, L.; BORRELL, C. y SUHRCKE, M. (2015). «Health and health behaviours before and during the Great Recession, overall and by socioeconomic status, using data from four repeated crosssectional health surveys in Spain (2001-2012)». BMC Public Health, 15 (1), 865.

[16] BARTOLL, X.; PALÈNCIA, L.; MALMUSI, D.; SUHRCKE, M. y BORRELL, C. (2014). «The evolution of mental health in Spain during the economic crisis». The European Journal of Public Health, 24, 415-418.

[17] BASTERRA, V. (2017). «Evolución de la prevalencia de alto riesgo de trastornos mentales en población adulta española: 2006-2012». Gaceta Sanitaria, 31 (4), 324-326.

[18] BAUMBACH, A. y GULIS, G. (2014). «Impact of financial crisis on selected health outcomes in Europe». The European Journal of Public Health, 24 (3), 399-403.

[19] BENMARHNIA, T.; ZUNZUNEGUI, M. V.; LLÁCER, A. y BÉLAND, F. (2014). «Impact of the economic crisis on the health of older persons in Spain: research clues based on an analysis of mortality». SESPAS report 2014. Gaceta Sanitaria, 28, 137-141.

[20] BLAKELY, T.; TOBIA, M. y ATKINSON, J. (2008). «Inequalities in mortality during and after restructuring of the New Zealand economy: repeated cohort studies». BMJ, 336, 371-375.

[21] BORRELL, C.; MARÍ-DELL'OLMO, M.; GOTSENS, M.; CALVO, M.; RODRÍGUEZSANZ, M.; BARTOLL, X. y ESNAOLA, S. (2017). «Socioeconomic inequalities in suicide mortality before and after the economic recession in Spain». BMC Public Health, 17, 772.

[22] BRENNER, M. H. (1979a). «Mortality and the national economy. A review, and the experience of England and Wales, 1936-76». Lancet, 314 (8142), 568-573.

[23] BRENNER, M. H. (1979b). «Unemployment, economic growth, and mortality». Lancet, 313 (8117), 672.

[24] BRENNER, M. H. (2005). «Commentary: economic growth is the basis of mortality rate decline in the 20th century-experience of the United States 1901-2000». Int. J. Epidemiol., 34 (6), 1214-1221.

[25] BUCHMUELLER, T.; FLORENCE, J. y GRIGNON M. (2007). Unemployment and Mortality in France, 1982-2002. Centre for Health Economics and Policy Analysis (CHEPA), McMaster University, Hamilton, Canadá.

[26] CALZÓN S.; FERNÁNDEZ, A.; LÓPEZ DEL AMO, M. D. P. y MARTÍN, J. J. (2017). «Diferencias por sexo de la salud percibida antes y durante la crisis económica (2007 y 2011)». Revista Española de Salud Pública, 91, 201702019.

[27] CASE, A. L. y PAXSON, C. (2002). «Economic Status and Health in Childhood: The Origins of the Gradient». American Economic Review, 92, 1308-1334. 
[28] CÓRDOBA-DOÑA J. A.; SAN SEBASTIÁN M.; ESCOLAR-PUJOLAR A.; MARTÍNEZ-FAURE J. E. y GUSTAFSSON, P. E. (2014). «Economic crisis and suicidal behaviour: the role of unemployment, sex and age in Andalusia, southern Spain». Int. J. Equity Health, 13 (1), 55.

[29] CÓRDOBA-DOÑA, J. A.; ESCOLAR-PUJOLAR, A.; SAN SEBASTIÁN, M. y GUSTAFSSON, P. E. (2016). «How are the employed and unemployed affected by the economic crisis in Spain? Educational inequalities, life conditions and mental health in a context of high unemployment». BMC Public Health, 16 (1), 267.

[30] COVENEY, M.; GARCÍA-GÓMEZ, P.; VAN DOORSLAER, E. y VAN OURTI, T. (2016). «Health disparities by income in Spain before and after the economic crisis». Health Economics, 25, 141-158.

[31] CUTLER, D.; KNAUL, F.; LOZANO, R.; MENDEZ, O. y ZURITA, B. (2000). «Financial crisis, health outcomes and aging: Mexico in the 1980s and 1990s». Journal of Public Economics, 84 (2), 279-303.

[32] CUTLER, D. M.; LLERAS-MUNEY, A. y VOGL, T. (2008). «Socioeconomic Status and Health: Dimensions and Mechanisms». National Bureau of Economic Research. Working Paper Series, 14333.

[33] DARMON, N. y DREWNOWSKI, A. (2008). «Does social class predict diet quality?». Am. J. Clin. Nutr., 87, 1107-17.

[34] DEATON, A. y PAXSON C. (2001). «Mortality, education, income, and inequality among American cohorts». Themes in the Economics of Aging. University of Chicago Press, 129-170.

[35] DREWNOWSKI, A. y SPECTER, S. E. (2004). «Poverty and obesity: the role of energy density and energy costs». Am. J. Clin. Nutr., 79, 6-16.

[36] EDWARDS, R. (2008). «Who is hurt by pro-cyclical mortality?». Soc. Sci. Med., 62, 2051-58.

[37] FERRANDO, J.; PALÈNCIA, L.; GOTSENS, M.; PUIG-BARRACHINA, V.; MARÍ-DELL'OLMO, M.; RODRÍGUEZ-SANZ, M.; BARTOLL, X. y BORRELL, C. (2018). «Trends in cancer mortality in Spain: the influence of the financial crisis». $G a$ ceta sanitaria.

[38] FLORES, M.; GARCÍA-GÓMEZ, P. y ZUNZUNEGUI, M. V. (2014). «Crisis económica, pobreza e infancia. ¿Qué podemos esperar en el corto y largo plazo para los "niños y niñas de la crisis"?». Informe SESPAS 2014. Gaceta Sanitaria, 28, 132-136.

[39] FORNELL, B.; CORREA, M.; DEL AMO, M. P. L. y MARTÍN, J. J. (2018). «Influence of changes in the Spanish labor market during the economic crisis (2007-2011) on perceived health». Quality of Life Research, 1-11.

[40] FOUNTOULAKIS, K. N.; KAWOHL, W.; THEODORAKIS, P. N.; KERKHOF A. J.; NAVICKAS, A. y HÖSCHL, C. (2014). «Relationship of suicide rates to economic variables in Europe: 2000-2011». Br. J. Psychiatry, 205 (6), 486-496.

[41] FRASQUILHO, D.; MATOS, M. G.; SALONNA, F.; GUERREIRO, D.; STORTI, C. C.; GASPAR,T.y CALDAS-DE-ALMEIDA,J.M.(2016). «Mental health outcomes in times of economic recession: a systematic literature review». BMC Public Health, 16 (1), 115.

[42] GERDTHAM, U. G. y RUHM, C. J. (2006). «Deaths rise in good economic times: evidence from the OECD». Economics \& Human Biology, (4), 3, 298-316.

[43] GOTSENS M.; MALMUSI D.; VILLRROEL N.; VIVES-CASES C.; GARCIASUBIRATS I.; HERNANDO, C. y BORREL C. (2015). «Health inequality between 
immigrants and natives in Spain: the loss of the healthy immigrant effect in times of economic crisis». Eur. J. Public Health, 25 (6), 923-929 .

[44] GILI, M.; ROCA, M.; BASU, S.; MCKEE, M. y STUCKLER, D. (2013). «The mental health risks of economic crisis in Spain: evidence from primary care centres, 2006 and 2010». The European Journal of Public Health, 23 (1), 103-108.

[45] GILI, M.; LÓPEZ-NAVARRO, E.; CASTRO, A.; HOMAR, C.; NAVARRO, C.; GARCÍA-TORO, M.; GARCÍA-CAMPAYO, J. y ROCA, M. (2016). «Gender differences in mental health during the economic crisis». Psicothema, 28 (4), 407-413.

[46] GONZÁLEZ LÓPEZ-VALCÁRCEL, B. y BARBER, P. (2017). «Economic crisis, austerity policies, health and fairness: lessons learned in Spain». Applied Health Economics and Health Policy, 15 (1), 13-21.

[47] GRAHAM, C. (2008). «Happiness and health: Lessons-and questions-for public policy». Health Affairs, 27 (1), 72-87.

[48] GROOSMAN, M. y KAESTNER, R. (1997). «Effects of education on health». En J. Behrman y N. Stacey (eds.), The Social Benefits of Education, 69-123. University of Michigan Press.

[49] GROOT, W. y MAASSEN VAN DEN BRINK, H. (2007). «The health effects of education». Economics of Education Review, 26, 186-200.

[50] HENARES-MONTIEL, J.; RUIZ-PÉREZ, I. y MENDOZA-GARCÍA, O. (2018). «Health inequalities between male and female immigrants in Spain after the beginning of the economic crisis». Health Soc. Care Community, 1-7.

[51] HERNÁNDEZ-QUEVEDO, C.; LÓPEZ-VALCÁRCEL, B. G. y PORTA, M. (2018). «Short-term adverse effects of austerity policies on mortality rates: what could their real magnitude be?». American Public Health Association, 983-985.

[52] HINTIKKA, J.; SAARINEN, P. I. y VIINAMAKI, H. (1999). «Suicide mortality in Finland during an economic cycle, 1985-1995». Scand. J. Public Health, 27, 85-88.

[53] IGLESIAS GARCÍA; SÁIZ MARTINEZ; GARCÍA-PORTILLA, M. P.; BOUSOÑO GARCÍA, M.; JIMÉNEZ TREVIÑO, L; SÁNCHEZ LASHERAS, F. y BOBES, J. (2014). «Efectos de la crisis económica en la demanda por trastornos mentales en Asturias: datos del Registro Acumulativo de Casos Psiquiátricos (2000-2010)». Actas Esp. Psiquiatr., 42 (3),108-15.

[54] JIN, R. L.; SHAH, C. P. y SVOBODA, T. J. (1995). «The impact of unemployment on health: a review of the evidence». CMAJ, 153 (5), 529-40.

[55] KARANIKOLOS, M.; MLADOVSKY, P.; CYLUS, J.; THOMSON, S.; BASU, S.; STUCKLER, D.; MACKENBACH, J. P. y MCKEE, M. (2013). «Financial crisis, austerity and health in Europe». Lancet, 381 (9874), 1323-1331.

[56] KHANG, Y. H.; LYNCH, J. W. y KAPLAN, G. A. (2005). «Impact of economic crisis on cause-specific mortality in South Korea». Int. J. Epidemiol., 34 (6), 1291-301.

[57] LAANANI, M.; GHOSN, W.; JOUGLA, E. y Rey, G. (2015). «Impact of unemployment variations on suicide mortality in Western European countries (2000-2010)». Journal Epidemiol. Community Health, 69 (2), 103-109.

[58] LEON, D. A.; CHENET, L.; SHKOLNIKOV, V. M.; ZAKHAROV, S.; SHAPIRO, J.; RAKHMANOVA, G.; VASSIN, S. y MCKEE, M. (1997). «Huge variation in Russian mortality rates 1984-94: artefact, alcohol or what?». Lancet, 350 (9075), 383-388.

[59] LEON, D. A.; SABUROVA, L.; TOMKINS, S.; ANDREEV, E.; KIRYANOV, N.; MCKEE, M. y SHKOLNIKOV, V. M. (2007). «Hazardous alcohol drinking and 
premature mortality in Russia: a population based case-control study». Lancet, 369 (9578), 2001-2009.

[60] LIBRERO, J.; SEGURA, A. y GONZÁLEZ LÓPEZ-VALCÁRCEL B. (2013). «Suicides, hurricanes and economic crisis». European Journal of Public Health, 24 (2), 183.

[61] LÓPEZ BERNAL, J. A.; GASPARRINI, A.; ARTUNDO, C. M. y MCKEE, M. (2013). «The effect of the late 2000s financial crisis on suicides in Spain: an interrupted timeseries analysis». The European Journal of Public Health, volumen 23, 732-736.

[62] LÓPEZ DEL AMO GONZÁLEZ, M. P.; BENÍTEZ, V. y MARTÍN-MARTÍN, J. J. (2018). «Long term unemployment, income, poverty, and social public expenditure, and their relationship with self-perceived health in Spain (2007-2011)». BMC Public Health, 18 (1), 133.

[63] MACHENBAN, J. P.; VALVERDE, J. R.; ARTNIK, B.; BOPP, M.; BRONNUM-HANSEN, H.; DEBOOSERE, P.; KALEDIENE, R.; KOVACS, K.; LEINSALU, M.; MARTIKAINEN, P.; REGIDOR, E.; RYCHTARIKOVA, J.; RODRÍGUEZ-SANZ, M.; VINEIR, P.; WHITE, C.; WOJTYNIAK, B.; HU, Y. y NUSSELDER, W. J. (2018). «Trends in health inequalities in 27 European countries». Proc. Natl. Acad. Sci. USA. Jun 19; 115 (25), 6440-6445.

[64] MACMAHON, B.; JOHNSON, S. y PUGH, T. F. (1963). «Relation of suicide rates to social conditions. Evidence from U.S. vital statistics». Public Health Reports, 78 (4), 285.

[65] MÁRQUEZ, S. (2012). Efectos de las crisis económicas en la salud y la equidad en salud: revisión de la literatura científica (mimeo, agosto).

[66] MÁRQUEZ-CALDERÓN, S.; RUIZ-RAMOS, M.; VICIANA, F. y FERNÁNDEZ MERINO, J. C. (2018). «Tendencia de la mortalidad edad y sexo específica en mayores de 60 años: España 1981-2016». XXXVI Reunión Científica de la SEE, 11-14 septiembre. Lisboa.

[67] MARTIN, M. (2002). «Substance use and social and economic transition: the need for evidence». International Journal of Drug Policy, 13 (6), 453-459.

[68] MAYNOU, L.; SÁEZ, M. y LÓPEZ-CASASNOVAS, G. (2014). «Has the economic crisis widened the intraurban socioeconomic inequalities in mortality? The case of Barcelona, Spain». J. Epidemiol. Community Health, jech-2013-203447.

[69] MEDEL-HERRERO, A. y GÓMEZ-BENEYTO, M. (2017). «The impact of the 2008 economic crisis on the increasing number of young psychiatric inpatients». Revista de Psiquiatria y Salud Mental. En prensa, corrected proof.

[70] MIRET, M.; CABALLERO, F. F.; HUERTA-RAMÍREZ, R.; MONETA, M. V.; OLAYA, B.; CHATTERJI, S. y AYUSO-MATEOS, J. L. (2014). «Factors associated with suicidal ideation and attempts in Spain for different age groups. Prevalence before and after the onset of the economic crisis». Journal of Affective Disorders, 163, 1-9.

[71] MONCHO, J.; PEREYRA-ZAMORA, P.; TAMAYO-FONSECA, N., GIRÓN, M.; GÓMEZ-BENEYTO, M. y NOLASCO, A. (2018). «Is recession bad for your mental health? The answer could be complex: evidence from the 2008 crisis in Spain». BMC Medical Research Methodology, 18 (1), 78.

[72] MURRAY, J. E. (2007). Origins of American Health Insurance: A History of Industrial Sickness Funds. Yale University Press, 2007.

[73] NEUMAYER, E. (2004). «Recessions lower (some) mortality rates: evidence from Germany». Soc. Sci.Med., 58, 1037-1047. 
[74] NOTZON, F. C.; KOMAROV, Y. M.; ERMAKOV, S. P.; SEMPOS, C. T.; MARKS, J. S. y SEMPOS, E. V. (1998). «Causes of declining life expectancy in Russia». JAMA, 279 (10), 793-800.

[75] ORTÚN RUBIO, V:; GONZÁLEZ LÓPEZ-VALCÁRCEL, B. y PINILLA, J. (2016). "El impuesto sobre bebidas azucaradas en España". Revista Española de la Salud Pública. volumen 90, pp.e1-e13 CE.

[76] OSTAMO, A. y LONNQVIST, J. (2001). «Attempted suicide rates and trends during a period of severe economic recession in Helsinki, 1989-1997». Society Psychiatry Epidemiol., 36, 354-60.

[77] RAJMIL, L.; MEDINA-BUSTOS, A.; DE SANMAMED, M. J. F. y MOMPART, A. (2013). «Impact of the economic crisis on children's health in Catalonia: A before-after approach». BMJ Open, 3 (8), e003286.

[78] RAJMIL, L.; SIDDIQI, A.; TAYLOR-ROBINSON, D. y SPENCER, N. (2015). «Understanding the impact of the economic crisis on child health: the case of Spain». International Journal for Equity in Health, 14, 1-9.

[79] REGIDOR, E.; BARRIO, G.; BRAVO, M. J. y DE LA FUENTE, L. (2014). «Has health in Spain been declining since the economic crisis?». Journal of Epidemiology and Community Health, 68, 280-282.

[80] REIBLING, N.; BECKFIELD, J.; HUIJTS, T.; SCHMIDT-CATRAN, A.; THOMSON, K. H. y WENDT, C. (2017). «Depressed during the depression: has the economic crisis affected mental health inequalities in Europe?». The European Journal of Public Health, 27 (1_1), 47-54.

[81] RIVADENEYRA-SICILIA, A.; MINUÉ-LORENZO, S. y ARTUNDO-PURROY, C. (2014). «Lecciones desde fuera. Otros países en esta y otras crisis anteriores». Gaceta Sanitaria. Informe SESPAS 2014, cap. 2.

[82] RIVERA, B.; CASAL, B. y CURRAIS, L. (2017). «Crisis, suicide and labour productivity losses in Spain». The European Journal of Health Economics, 18, 83-96.

[83] RODRÍGUEZ-CARO, A.; VALLEJO-TORRES, L.; LÓPEZ-VALCÁRCEL, B. (2016). «Unconditional quantile regressions to determine the social gradient of obesity in Spain 1993-2014». International Journal for Equity in Health, 15, 1, 175.

[84] RUHM, C. J. (2000). «Are recessions good for your health?». Quarterly Journal of Economics, 115 (2), 617-650.

[85] RUHM, C. J. (2005). «Healthy living in hard times». Journal of Health Economics, 24 (2), 341-363.

[86] RUHM, C. J. (2015). «Recessions, Healthy No More?». Journal of Health Economics, 42, 17-28.

[87] RUIZ-PÉREZ, I.; BERMÚDEZ-TAMAYO, C. y RODRÍGUEZ-BARRANCO, M. (2017). «Socio-economic factors linked with mental health during the recession: a multilevel analysis». International Journal for Equity in Health, 16 (1), 45.

[88] RUIZ-RAMOS, M.; CÓRDOBA-DOÑA, J. A.; BACIGALUPE, A.; JUÁREZ, S. y ESCOLAR-PUJOLAR, A. (2014). «Crisis económica al inicio del siglo xxI y mortalidad en España. Tendencia e impacto sobre las desigualdades sociales». Gaceta Sanitaria. Informe SESPAS 2014, 28, 89-96.

[89] SAURINA C.; MARZO, M. y SÁEZ, M. (2015). «Inequalities in suicide mortality rates and the economic recession in the municipalities of Catalonia, Spain». International Journal for Equity in Health, 14 (1), 1-10. 
[90] SEGURA, A. (2014). «Recortes, austeridad y salud». Gaceta Sanitaria. Informe SESPAS, suplemento 1 , capítulo 1 .

[91] SHKOLNIKOV, V.; MCKEE, M. y LEON, D. A. (2001). «Changes in life expectancy in Russia in the mid-1990s». Lancet, 357 (9260), 917-921.

[92] SICRAS-MAINAR, A. y NAVARRO-ARTIEDA, R. (2016). «Use of antidepressants in the treatment of major depressive disorder in primary care during a period of economic crisis». Neuropsychiatric Disease and Treatment, 12, 19.

[93] STIGLITZ, J. (1999). «Responding to economic crises: policy alternatives for equitable recovery and development». The Manchester School, 67, 409-427.

[94] STUCKLER, D. y BASU, S. (2013). «The body economic: Eight experiments in economic recovery, from Iceland to Greece». Penguin UK.

[95] STUCKLER, D.; BASU, S.; SUHRCKE, M.; COUTTS, A. y MCKEE, M. (2009a). «The public health effect of economic crises and alternative policy responses in Europe: an empirical analysis». The Lancet, 374 (9686), 315-323.

[96] STUCKLER, D.; KING, L. y MCKEE, M. (2009b). «Mass privatisation and the postcommunist mortality crisis: a cross-national analysis». Lancet, 373 (9661), 399-407.

[97] STRUMPF, E. C.; CHARTERS, T. J.; HARPER, S. y NANDI, A. (2017). «Did the Great Recession affect mortality rates in the metropolitan United States? Effects on mortality by age, gender and cause of death». Social Science \& Medicine, 189, 11-16.

[98] SYDENSTRICKER, E. (1934). «Health in the New Deal». Annals of the American Academy of Political and Social Science, 176 (1), 131-137.

[99] TANGCHAROENSATHIEN, V.; HARNVORAVONGCHAI, P.; PITAYARANGSARIT, S. y KASEMSUP, V. (2000). «Health impacts of rapid economic changes in Thailand». Soc. Sci.Med., 51, 789-807.

[100] TAPIA GRANADOS, J. A. (2005a). «Increasing mortality during the expansions of the US economy, 1900-1996». Int. J. Epidemiol., 34, 1194-202.

[101] TAPIA GRANADOS, J. A. (2005b). «Recessions and Mortality in Spain, 1980-1997». Journal of Population, 21, 393-422.

[102] TAPIA GRANADOS, J. A. y IONIDES, E. L. (2017). «Population health and the economy: Mortality and the Great Recession in Europe». Health Economics, 26, e219-e235.

[103] TOM, B.; MICHEL, G. y FLORENCE, J. (2007). Unemployment and Mortality in France, 1982-2002. Centre for Health Economics and Policy Analysis (CHEPA), McMaster University, Hamilton, Canadá.

[104] URBANOS, R. y GONZÁLEZ LÓPEZ-VALCÁRCEL, B. (2013). «Desempleo y salud: un análisis de la repercusión de la crisis económica sobre la salud de los españoles». Rev. Estudios de Economia Aplicada, 31 (2).

[105] VALLEJO-TORRES,L.; MORRIS,S.y LÓPEZ-VALCÁRCEL,B. G. (2018). «Obesity and perceived work discrimination in Spain». Applied Economics, 50 (36), 3870-3884.

[106] VALKONEN, T.; MARTIKALNENU, P.; JALOVAARA, M.; KCAKINEN, S.; MARTELLN, T. y MAKEIA, P. (2000). «Changes in socioeconomic inequalities in mortality during an economic boom and recession among middle-aged women in Finland». Eur. J. Public Health, 10, 274-280.

[107] VÁZQUEZ, M.; VARGAS, I. y ALLER, M. (2014). «Reflexiones sobre el impacto de la crisis en la salud y la atención sanitaria de la población inmigrante». Gaceta Sanitaria. Informe SESPAS 2014, suplemento 1, capítulo 22. 
[108] WADA, K.; KONDO, N.; GILMOUR, S.; ICHIDA, Y.; FUJIMO, Y.; SATOH, T. y SHIBUYA, K. (2012). «Trends in cause specific mortality across occupations in Japanese men of working age during period of economic stagnation, 1980-2005: retrospective cohort study». BMJ, 344, e1191.

[109] WALBERG, P.; MCKEE, M.; SHKOLNIKOV, V.; CHENET, L. y LEON, D. A. (1998). «Economic change, crime, and mortality crisis in Russia: regional analysis». BMJ, 317 (7154), 312-318.

[110] WATERS, H.; SAADAH, F. y PRADHAN, M. (2003). «The impact of the 1997-98 East Asian economic crisis on health and health care in Indonesia». Health Policy Plan, 18, 172-181.

[111] ZAPATA MOYA A.; BUFFEL V.; NAVARRO YÁÑEZ, C. y BRACKE, P. (2015). «Social inequality in morbidity, framed within the current economic crisis in Spain». International Journal for Equity in Health, 14 (1), 131. 